\title{
Application of XMR 2D-3D Registration to Cardiac Interventional Guidance
}

\author{
Kawal S. Rhode ${ }^{1}$, Derek L. G. Hill ${ }^{1}$, Philip J. Edwards ${ }^{1}$, John Hipwell ${ }^{1}$, \\ Daniel Rueckert ${ }^{2}$, Gerardo I. Sanchez-Ortiz ${ }^{2}$, Sanjeet Hegde ${ }^{1}$, Vithuran Rahunathan ${ }^{1}$, \\ and Reza Razavi ${ }^{1}$ \\ ${ }^{1}$ Division of Imaging Sciences, King's College London, UK. \\ ${ }^{2}$ Department of Computer Sciences, Imperial College London, UK.
}

\begin{abstract}
We describe a registration and tracking technique to integrate cardiac $\mathrm{x}$-ray images and cardiac magnetic resonance (MR) images acquired from a combined $\mathrm{x}$-ray and MR interventional suite (XMR). Optical tracking is used to determine the transformation matrices relating MR and $\mathrm{x}$-ray image coordinates. Calibration and tracking enable us to combine $\mathrm{x}$-ray projection images with registered projection MR images from a volume acquisition, and to display $3 \mathrm{D}$ reconstructions of catheters within the MR volume. Registration errors were assessed using phantom experiments. Errors in the combined projection images (2D root mean square target registration error - RMS TRE) were found to be $2.4-4.2 \mathrm{~mm}$, and the errors in the integrated volume representation (3D RMS TRE) were found to be $4.6-5.1 \mathrm{~mm}$. These errors are clinically acceptable for alignment of images of the heart and the great vessels. We demonstrate that our technique can be used to guide catheters and to combine electrical and mechanical information in the context of the patient-specific anatomy for a patient undergoing cardiac radio-frequency $(\mathrm{RF})$ ablation.
\end{abstract}

\section{Introduction}

XMR suites are interventional facilities that incorporate both MR and $\mathrm{x}$-ray imaging [1-2]. These suites have two capabilities. Firstly, they provide a migration path so that interventions traditionally done under $\mathrm{x}$-ray guidance can be carried out under MR guidance, with the reassurance of X-ray backup. Secondly, XMR suites offer the potential to carry out interventions that benefit from the mixture of MR and X-ray for guidance. For both capabilities, it is desirable to be able to combine the images from $\mathrm{X}$-ray and MR for use during interventions, and also for retrospective analysis. The XMR suite at Guy's hospital is used for diagnostic cardiac catheterization of patients with congenital heart disease - where MRI can be used for the entire procedure - and for combined MR and X-ray guided cardiac interventions. In this paper we describe a registration and tracking technique that we have devised for use during XMR procedures. This provides two capabilities: overlay of projections calculated from the 3D MR images on the X-ray views, and display of 3D reconstructed structures from biplane $\mathrm{x}$-ray in $3 \mathrm{D}$ MR images. Our technique is demonstrated on phantoms and images from a patient undergoing cardiac RF ablation. 


\section{Method}

\subsection{Description of XMR Facility}

The XMR interventional suite at King's College London comprises an X-ray and RF shielded interventional room containing a 1.5T cylindrical bore MR scanner (Philips Intera I/T) and a mobile cardiac X-ray set (Philips BV Pulsera). The patient can be easily moved between the two systems using a specially modified sliding MR table top that docks with and transfers patients to a specially modified x-ray table (Philips Angio Diagnost 5 Syncratilt table). The docking and transfer takes less than 60 seconds.

\subsection{Modifications for Tracking and Registration}

Registration of XMR images requires finding the transformation that maps $3 \mathrm{D}$ points in MR image space to $2 \mathrm{D}$ points in $\mathrm{X}$-ray image space. This is achieved by a combination of system calibration and tracking. It is necessary to track the three moving components of the XMR system. The sliding MR table top is automatically tracked by the MR scanner when docked with the MR table. The X-ray C-arm and the X-ray table each have 6 infrared emitting diodes (IREDs) affixed, which are tracked by a Northern Digital Optotrak 3020.

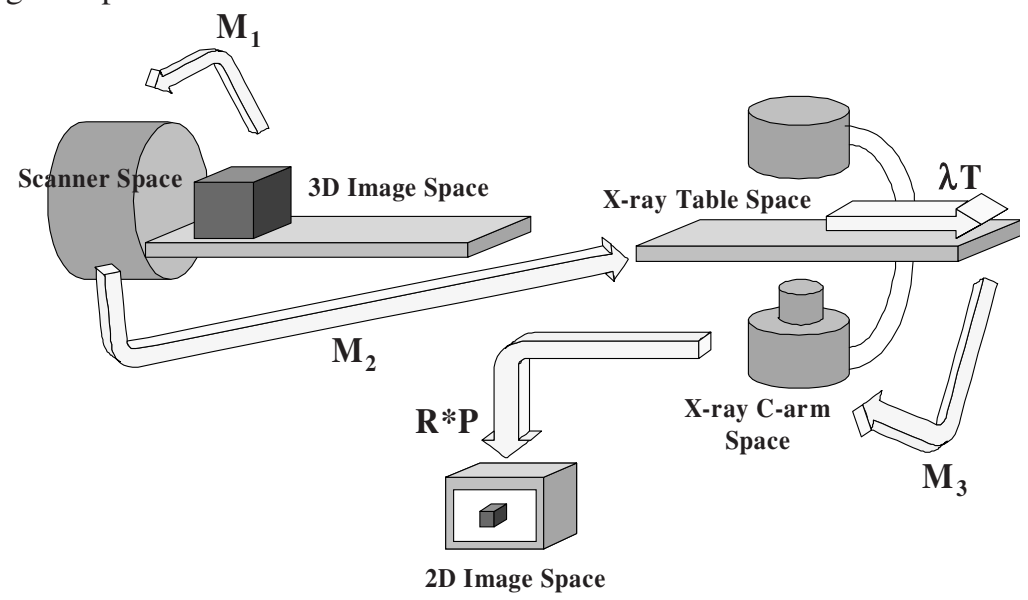

Fig. 1. Relationship between coordinate systems and transformation matrices.

The relationship between the coordinate systems is shown in figure 1. For the two $\mathrm{X}$-ray components a rigid body transformation matrix is calculated that maps points from X-ray C-arm space and X-ray table space to Optotrak space. Let $\mathbf{X}_{C}$ be the transformation from X-ray C-arm space to Optotrak space, and let $\mathbf{X}_{T}$ be the transformation from $\mathrm{x}$-ray table space to Optotrak space. Then

$$
\mathbf{M}_{3}=\mathbf{X}_{C}^{-1} \mathbf{X}_{T} \text {. }
$$




\subsection{Calibration of X-ray Perspective Geometry}

During cardiac catheterisation, $\mathrm{x}$-ray images are acquired from many different views, and the perspective projection changes with view due to non-rigidity of the x-ray gantry and changes in local magnetic field. This makes calibration from a single view insufficiently accurate. We therefore devised a novel technique to calculate an "average" projection matrix based on multiple views. The perspective projection calibration process involves acquisition of multiple $\mathrm{x}$-ray views of an acrylic calibration object that incorporates 14 point markers. The markers are based on those used in the Acustar neurosurgical guidance system [3]. The calibration object needed to be imaged with both MR imaging and X-ray imaging without being moved with respect to the sliding table top. Initially the table top was docked to the x-ray table and the position of the markers was located in x-ray table space using an Optotrak pointing device. Then 12 tracked $\mathrm{x}$-ray images were acquired with the $\mathrm{x}$-ray gantry being moved to cover the typical locations used for interventions. The 2D position of 48 markers that were in the field of view was found. The corresponding 3D positions were calculated by transforming the previously determined marker positions in x-ray table space to $\mathrm{C}$-arm space using the matrix $\mathbf{M}_{3}$ for each x-ray image. Now,

$$
\mathbf{P C}_{3 D}=\mathbf{C}_{2 D} \text {, }
$$

where $\mathbf{C}_{3 D}$ is the matrix of $3 \mathrm{D}$ point coordinates, $\mathbf{C}_{2 D}$ is the matrix of corresponding $2 \mathrm{D}$ point coordinates, and $\mathbf{P}$ is the perspective projection matrix. Equation (2) leads to an over determined set of linear equations and $\mathbf{P}$ was then found using singular value decomposition.

\subsection{Calibration of the Transformation between 3D Image Space and X-ray Table Space}

It is necessary to determine the transformation between MR scanner space and the $\mathrm{x}$ ray table space (matrix $\mathbf{M}_{2}$ ). To determine this transformation, the sliding table top is docked with the MR scanner and the calibration object is imaged (T1-weighted volume MR scan, 256x256 matrix, 200 slices, resolution $=1.05 \times 1.05 \times 1.2 \mathrm{~mm}$, $\mathrm{TR}=14.0, \mathrm{TE}=6.0$ ). The 3D position of the markers is determined in MR image space. Now,

$$
\mathbf{F}_{X R T}=\mathbf{M}_{2} \mathbf{M}_{1} \mathbf{F}_{I},
$$

where $\mathbf{F}_{X R T}$ is the matrix of marker locations in x-ray table space, $\mathbf{M}_{1}$ is the transformation from 3D image space to scanner space (determined from image header information), and $\mathbf{F}_{I}$ is the matrix of marker locations in 3D image space. Equation (3) is solved to compute $\mathbf{M}_{2}$, the transformation from scanner space to x-ray table space. The overall transformation from 3D image space to $\mathrm{x}$-ray table space must also include a variable translation parallel to the axis of motion of the sliding table top. The unit translation $\mathbf{T}$ is calculated by moving the calibration object along the sliding table top parallel to its axis of motion and marking the position of markers using the 
Optotrak pointing device. The magnitude of the translation $\lambda$ is determined from the tracking of the sliding table top provided by the MR scanner software.

\subsection{Generation of the Overall Registration Matrix}

Once the calibration steps have been carried out it is then possible to generate a $3 \mathrm{D}$ to $2 \mathrm{D}$ registration matrix for any $\mathrm{MR}$ scans and tracked $\mathrm{x}$-ray images. The matrices $\mathbf{M}_{2}, \mathbf{T}$, and $\mathbf{P}$ are determined from the calibration, whereas $\mathbf{M}_{1}, \mathbf{M}_{3}$, and the parameter $\lambda$ are specific to the MR acquisition and the tracked $x$-ray images. Furthermore, since the radiographer is allowed to arbitrarily rotate the x-ray image for correct anatomical viewing, the $2 \mathrm{D}$ rotation matrix $\mathbf{R}$ needs to be determined by marking corresponding points in $2 \mathrm{D}$ images acquired pre- and post-rotation. The overall registration matrix is then given by

$$
\mathbf{W}=\mathbf{R P M}_{3} \lambda \mathbf{T} \mathbf{M}_{2} \mathbf{M}_{1} .
$$

\subsection{Validation with Point-Based Test Object}

The first validation experiment was carried out using the calibration object described above as a phantom. The phantom was imaged using a T1-weighted volume MR scan (256x256 matrix, 200 slices, resolution $=1.05 \times 1.05 \times 1.2 \mathrm{~mm}, \mathrm{TR}=14.0, \mathrm{TE}=6.0)$. The table top was then docked to the $\mathrm{x}$-ray system, and five biplane tracked $\mathrm{x}$-ray images were acquired. The registration matrix was calculated for each image and this was used to generate maximum intensity projections (MIPs) through the MR volume. The $2 \mathrm{D}$ registration error was quantified by marking the location of the imaging markers in both the X-ray images and the MIPs. In total 35 markers where located in 2D. The $3 \mathrm{D}$ error was found by finding the position of the markers from the biplane $\mathrm{x}$-ray views using the epipolar constraint [4] and by manual marking in the MR volume scan. In total 17 markers where located in 3D.

\subsection{Validation with Anthropomorphic Phantom}

The second set of validation experiments were carried out using an anthropomorphic vascular phantom. This incorporates a silicone model of one half of the circle of Willis. It was connected to a pulsatile flow pump that circulated saline with an average flow of approximately $450 \mathrm{ml}$ per minute. Nine MR imaging markers were placed on the vascular phantom to assess the registration error. The phantom was imaged using a phase contrast MR sequence (256x256 matrix, 110 slices, resolution $=0.86 \times 0.86 \times 0.86 \mathrm{~mm}, \mathrm{TR}=9.2, \mathrm{TE}=4.3)$ and a T1-weighted volume MR sequence $(512 \times 512$ matrix, 190 slices, resolution $=0.43 \times 0.43 \times 0.8 \mathrm{~mm}, \mathrm{TR}=7.3, \mathrm{TE}=2.0)$. It was then transferred to the $\mathrm{x}$-ray system and tracked dynamic images were acquired from two oblique views during the injection of contrast medium. Using the registration matrices and the epipolar constraint it is possible to semi-automatically track vessels from the two $\mathrm{x}$-ray views [4]. This provides a 2D centreline representation for each $\mathrm{X}$ ray view, together with a single $3 \mathrm{D}$ centreline representation for each pair of views. 
This was carried out for the internal carotid artery segment of the vascular phantom. The registration matrices were used to generate MR MIPs corresponding to the x-ray projections. The $2 \mathrm{D}$ centrelines of the vessels in these MIPs, and the corresponding $3 \mathrm{D}$ centreline could be calculated using the same algorithm used for the X-ray images. A $2 \mathrm{D}$ registration error was also calculated from the location of the imaging markers in the X-ray images and the MR MIPS. A total of 12 markers were visible in the pair of x-ray images. The TRE was quantified in $2 \mathrm{D}$ and $3 \mathrm{D}$ using the centreline representation above.

\subsection{Clinical Validation}

The system was calibrated prior to the patient investigation using the technique described in sections 2.3 and 2.4 above. The patient (male, aged 15) had an intermittent ventricular tachycardia that was to be treated by electrophysiology study and RF ablation. During the MR part of the procedure, MR scans of the heart and the great vessel were acquired using an SSFP three-dimensional multiphase sequence (3 phases, $256 \times 256$ matrix, 128 slices, resolution=1.13x1.13x1.0mm, TR=3.1, TE=1.6). During the $\mathrm{x}$-ray part of the procedure, tracked biplane $\mathrm{x}$-ray images were acquired. Registration matrices were calculated for each of the tracked views. Additionally for this patient, tagged MR sequences $(256 \times 256$ matrix, 59 phases, resolution $=1.33 \times 1.33 \times 8.0 \mathrm{~mm}$, $\mathrm{TR}=11.0, \mathrm{TE}=3.5$, tag spacing $=8 \mathrm{~mm}$ ) were also acquired from which the myocardial motion was quantified using a non-rigid registration technique [5]. The registration enabled us to relate the position of the measured electrophysiology data to the cardiac motion.

\section{Results}

\subsection{Calibration}

The robustness of the projection matrix calculation was assessed using a leave-oneout experiment. The 2D RMS error between the position of markers located in the $\mathrm{x}$ ray images and the position of markers calculated using the perspective projection matrix was found to be $2.0 \mathrm{~mm}(\mathrm{n}=48)$.

\subsection{Validation with Point-Based Test Object}

2D RMS TRE calculated from the location of the markers in the x-ray images and the MR MIPs generated using the registration matrices was found to be $4.2 \mathrm{~mm}(n=35)$. The 3D RMS TRE calculated from the location of markers MR volume and the pairs of x-ray views was found to be $4.6 \mathrm{~mm}(\mathrm{n}=17)$. 


\subsection{Validation with Anthropomorphic Phantom}

Figure 2a shows one of the two x-ray images of the vascular phantom with the MR MIP overlay generated using the registration matrix. The 2D RMS TRE for the target vessel centreline was found to be $2.7 \mathrm{~mm}(\mathrm{n}=255)$ for the first view and $2.4 \mathrm{~mm}$ $(n=242)$ for the second view. The 3D RMS TRE was found to be $5.1 \mathrm{~mm}(\mathrm{n}=233)$ computed from both views. The 2D RMS TRE computed using the imaging markers was $3.6 \mathrm{~mm}(\mathrm{n}=12)$.

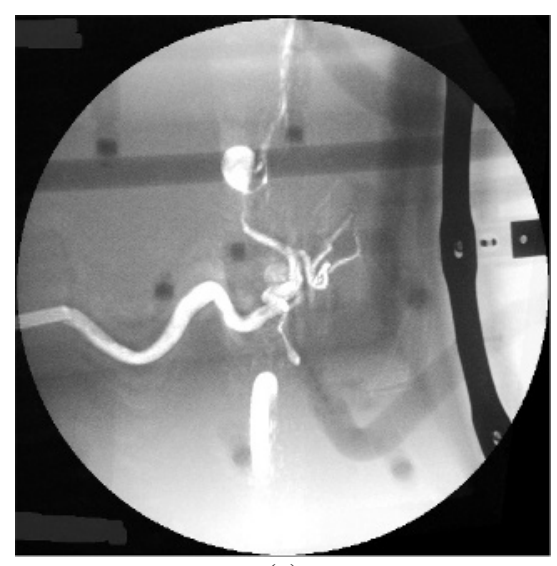

(a)

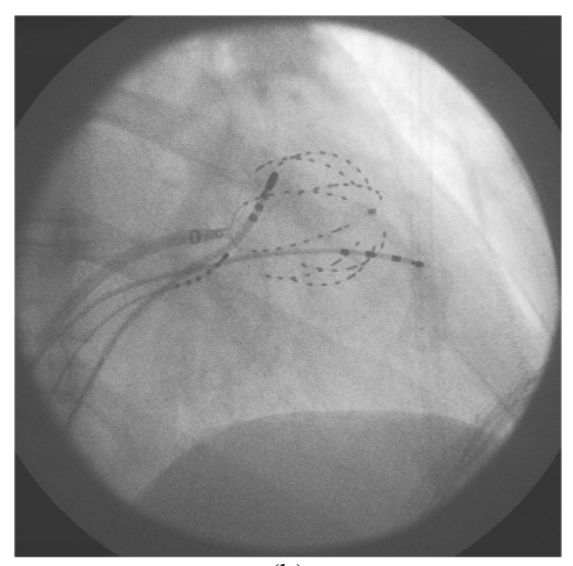

(b)

Fig. 2. (a) One of two x-ray views of the vascular phantom with the MR MIP overlaid. (b) One of the two x-ray views from the patient data showing 3 electrical measurement catheters and one electrode basket catheter in the right ventricle.

\subsection{Clinical Validation}

Figure $2 \mathrm{~b}$ shows one of the biplane $\mathrm{x}$-ray views acquired during the electrophysiology study for this patient. Four catheters can be seen in place. Three of these are measurement catheters with several electrodes along their lengths, and the other is a basket catheter with 67 electrodes distributed approximately over a spherical surface. Figure 3 shows the MR-derived surface rendering of the right side of this patient's heart. Added to this are the three measurement catheters reconstructed using the registered biplane x-ray views and the basket catheter shown as a sphere whose centre and diameter were found from the registered views. Also shown is an example of the electrograms recorded along one of the splines of the basket catheter. Figure 4 shows renderings of the electrode basket, the tagged MR images and the derived myocardial motion in the same coordinate system. The motion vectors were derived from the dynamic tagged images using non-rigid registration. 

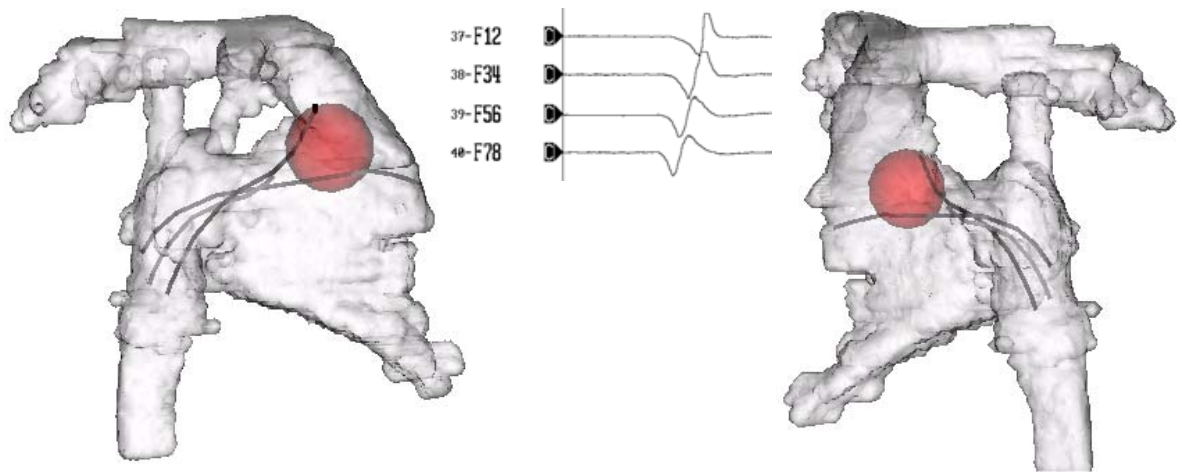

Fig. 3. (Left \& Right) Surface rendering of the right side of the heart derived from the MR images incorporating the three reconstructed catheters and the basket catheter shown as a sphere. (Centre) Electrogram recordings showing the progression of depolarization along one of the splines in the basket catheter.
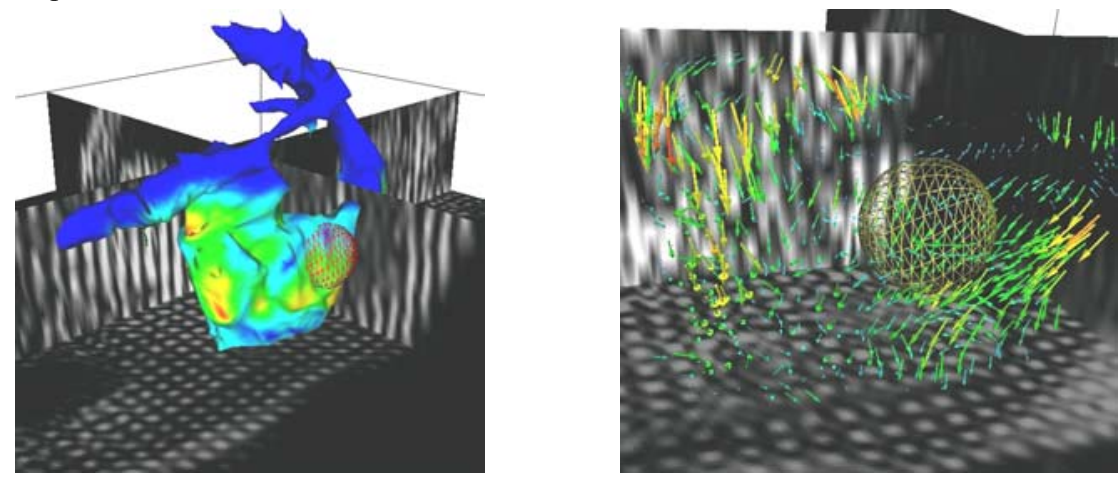

Fig. 4. The electrode basket, tagged MR image slices, and motion-encoded heart surface (Left), and derived motion vectors (Right) all displayed in the same coordinate system.

\section{Discussion and Conclusions}

XMR systems, in which MRI and x-ray imaging are available in the same room, allow endovascular procedures to be carried out under a combination of MR and X-ray guidance. We have described our novel approach for the registration of these imaging modalities to provide an integrated representation of the patient for use during guidance. Our technique determines the relative positions of the x-ray set, patient and MR scanner using a combination of IREDs tracked by an Optotrak, and the MR scanner's own table tracking system. Having carried out an initial calibration stage, this tracking information enables us to generate a projection from an MR image to match the current $\mathrm{x}$-ray view, and also to reconstruct the 3D position of a point or line from a pair of x-ray views, and transfer this 3D structure into MR coordinates.

The lack of visualisation of the heart and great vessels during x-ray-guided cardiac catheterisation is a real disadvantage especially during interventional procedures such 
as cardiac RF ablation. This leads to prolongation of the procedure time, increased $\mathrm{x}-$ ray radiation dose and is often the main cause for unsuccessful outcome and the need for repeat cardiac catheterisation. There is a relationship between the electrical activation and motion of the myocardium. We can measure the motion by processing tagged MR images and our registration method allows us to relate the location of the measurement electrodes positioned under x-ray guidance to the MR-derived motion. In the future this opens up a new avenue of research and as well as providing extra information for the cardiologist during the ablation procedure it has the potential of replacing part of the invasive $\mathrm{x}$-ray-guided cardiac electrophysiology study with non-invasive MR-guidance.

Our phantom results show that the potential accuracy of the system is approximately $4 \mathrm{~mm}$. Patient motion during a procedure will reduce this accuracy. The patients investigated in our centre all have a general anaesthetic and paralysis. This reduces the likelihood of the patient moving substantially on the table, and also enables us to overcome respiratory motion by acquiring the images with a ventilatorcontrolled end-expiration image acquisition. We aim to improve accuracy by better synchronisation with the cardiac cycle, application of MR and X-ray geometric distortion correction, and refinement of the registration transformation using an intensity registration algorithm [6-7].

\section{References}

1. R. Fahrig, K. Butts, J. A. Rowlands, R. Saunders, J. Stanton, G. M. Stevens, B. L. Daniel, Z. Wen, D. L. Ergun, N. J. Pelc, “A truly hybrid interventional MR/x-ray system: feasibility demonstration,” J. Magn. Reson. Imaging, 13(2), pp. 294-300, 2001.

2. G. Adam, J. Neuerburg, A. Buecker, A. Glowinski, D. Vorwerk, A. Stargardt, J. J. VanVaals, R. W. Gunther, "Interventional magnetic resonance - initial clinical experience with a 1.5-tesla magnetic resonance system combined with c-arm fluoroscopy," Investigative Radiology, 32 (4), pp. 191-197, 1997.

3. C. R. Maurer Jr., J. M. Fitzpatrick, M. Y. Wang, R. L. Galloway Jr, R. J. Maciunas, G. S. Allen, "Registration of head volume images using implantable fiducial markers," IEEE Trans. Med. Imag. 16(4), pp. 447-62, 1997.

4. D. J. Hawkes, A. C. F. Colchester, C. Mol, "The accurate 3D reconstruction of the geometric configuration of vascular trees from x-ray recordings," In Physics and Engineering of Medical Imaging, Guzzardi, Ed. The Hague: Martinus Nijhoff, 1985.

5. R. Chandrashekara, R. H. Mohiaddin, D. Rueckert, "Analysis of myocardial motion in tagged MR images using non-rigid image registration," In Proc. SPIE Medical Imaging, vol. 4684, pp. 1168-1179, 2002.

6. G. P. Penney, J. Weese, J. A. Little, P. Desmedt, D. L. G. Hill, D. J. Hawkes, “A comparison of similarity measures for use in 2D-3D medical image registration", IEEE Trans. Med. Imag., 17, pp. 586-595, 1998

7. J. H. Hipwell, G. P. Penney, T. C. S. Cox, J. V. Byrne, D. J. Hawkes, "2D-3D intensity based registration of DSA and MRA - a comparison of similarity measures," in Proc. MICCAI, Springer Lecture Notes in Computer Science, vol. 2489, pp. 501-508, 2002. 